\title{
A method of iris tracking based on machine vision
}

\author{
LI dong-shuang \\ College of Information Science and Technology, \\ Northwest University, Xi'an 710127 \\ lidongshuanglds@163.com
}

\author{
ZHONG lan-xiang \\ College of Information Science and Technology, \\ Northwest University, Xi'an 710127
}

\begin{abstract}
Through analyzing the advantages and disadvantages of the template matching method, Kalman Filter and Mean Shift algorithm, we proposed the method of combining them. We use the template matching method to initialize the irises positions, extract the eyes area, and then combined Kalman filter with Mean Shift algorithm to position and track irises accurately. The method also added to the average speed of the targets, it not only could be used as the standard if the irises are affected by the background, but also could be used as the irises speed when eyes are blocked, and then forecast the goals possible area in the next frame. This method is able to overcome the problems about irises tracking failure when there are tilt angle, deflection angle and pitch angle of the head in a certain range, and the object occlusion and background interference problems, which compressed the amount of computation and improved robustness of the iris tracking.
\end{abstract}

Keywords-iris tracking, template matching, Kalman filter, Mean Shift algorithm

\section{INTRODUCTION}

The voice technology which replaces or partially replaces the mouse and keyboard inputs for human-computer interaction has entered a practical stage currently. The technology of the iris tracking and input based on machine vision receives the attention of researchers and enterprises increasingly. Using iris tracking could get the direction of the target and aim it, and even develop intelligent computer and virtual games. In addition, the eye state detection and sight tracking could also effectively reflect the mood, mental and prevent the fatigue driving. However, the iris detection and tracking research is still in the initial stage at home and abroad, the literatures and the achievements are rarely. The improved template matching method compressed the calculation and shortened the run time ${ }^{[1]}$, but when the head deflection angle or pitch angle is bigger, the iris image would be distorted, affects the template matching seriously. Especially in the blinking, the iris is blocked, which results in the failure of template matching. The combination of Kalman filter and Mean Shift method is the moving target tracking method, it can effectively resolve the problems mentioned above. But the background influences the method seriously, and adjacent two frame correlation, if a frame appear tracking error, the position in the next frame is likely to be wrong ${ }^{[2,3]}$.

In order to overcome these difficulties, this paper proposes a combination of template matching, Kalman Filter and Mean Shift algorithm. This method uses the template matching method to position the iris center coordinates firstly. In order to avoid affecting the tracking results due to the color characteristics of the hair or the eye similar to the background, we intercepted the image of eye. Then we use the Kalman filter to predict the eye position in the intercepted image and calculate the covariance. Finally, according to the distribution characteristics of the eye brightness, we use Mean Shift iterative algorithm search the target in the estimated domain. After complex calculations, the center position of the target is the coordinates of the iris.

\section{INITIALIZE IRIS POSITION}

First of all, we use a digital camera to obtain the color image of the human face as shown in the figure 1(a). Then it is converted into the gray image. We chose the threshold which could highlight eyes' feature the most is 58, use global threshold method to extract eyes' area, the results are shown in the figure 1(b). Because the image is a face image, the irises will not close to the image edge, we can eliminate the edge area, such as hair and other not interested regions, only keep eyes area by the method of background removal, as shown in the figure 1(c). Finally, to highlight the irises' region, we abate the noise which generated by relative motion between the visual system and environment, distortion of the optical system and so on, and enhance the contrast between the irises and the surrounding area. After the fill hollow and smooth filter, we get the figure 1(d).

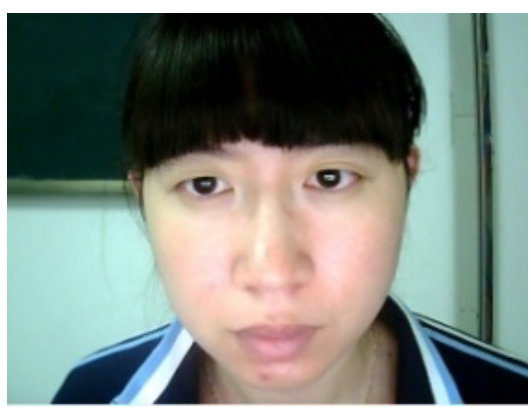

(a) Original image 


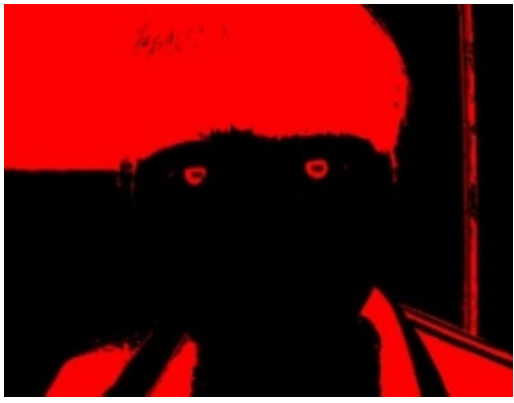

(b) Threshold segmentation results

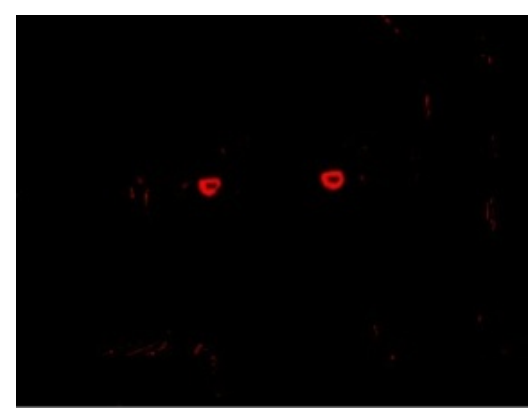

(c) Eliminate edge results

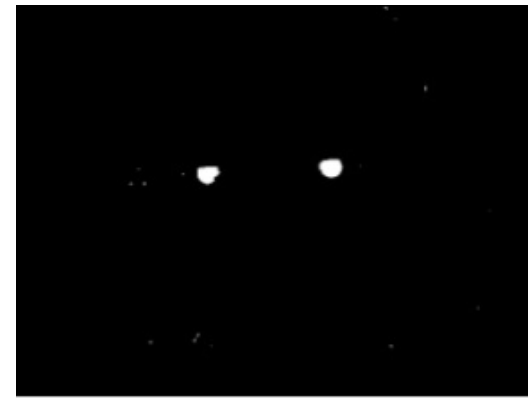

(d) Final results

Figure 1 Image preprocessing results

The irises position obtained by the above process are not the actual irises center position, so we also need to use the template matching method positioning irises coordinates accurately. Firstly, we extract the iris template from the standard image. According to the similarity of the template and each part of the image, it takes the center positions of the two objects in the image as the left and right iris coordinates respectively, as shown in figure 2. It averages coordinates of the previous $\mathrm{N}$ frames, and as the center of this value, intercepts a rectangular eye region image to be the original image of the next step on 400 pixels long and 60 pixels wide, as shown in the figure 3 .

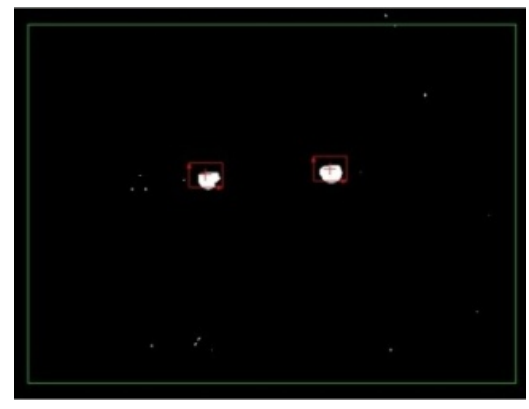

Figure 2 Iris initial positioning

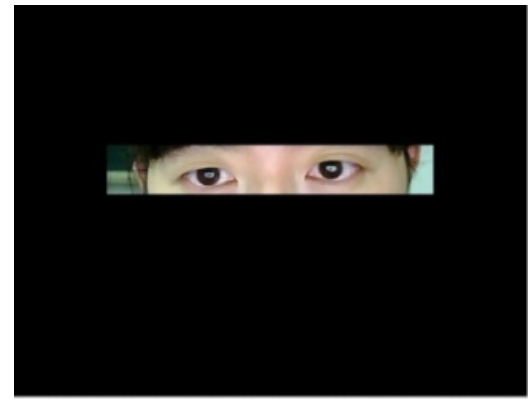

Figure 3 Area of the eye image

III. IRIS PRECISE POSITIONING AND TRACKING

Assumed that the target coordinates of the center in the image is $y_{0}$, each pixel of the target area is $\left\{x_{i}\right\} i=1, \ldots, n$. Firstly, we convert the image to the HSV image, because the $\mathrm{H}$ color vector of the type of image affected by light rarely. Using $\mathrm{H}$ vector to establish color histogram, expressed as:

$$
\hat{q}_{u}=C \sum_{i=1}^{n} k\left(\left\|\frac{y_{0}-x_{i}}{h}\right\|^{2}\right) \delta\left[b\left(x_{i}\right)-u\right] \quad i=1,2 \ldots . . m
$$

Among them, $k(x)$ is used to reduce influence of the target edge color by environmental factors, $h$ is bandwidth of kernel function, $b\left(x_{i}\right)$ is color index of $x_{i}, C$ is normalized coefficient.

The candidate target in the next frame is expressed as:

$$
\hat{p}_{u}(y)=C_{h} \sum_{i=1}^{n_{k}} k\left(\left\|\frac{y-x_{i}}{h}\right\|^{2}\right) \delta\left[b\left(x_{i}\right)-u\right] \quad i=1,2 \ldots . . m
$$

The target tracking can be simplified to search for the optimal $y$, makes $\hat{q}_{u}$ and $\hat{p}_{u}(y)$ is the most similar, the Bhattacharyya coefficient $\hat{\rho}(y)$ represent the similarities between them,

$$
\hat{\rho}(y)=\rho(\hat{p}(y), \hat{q})=\sum_{u=1}^{m} \sqrt{\hat{p}_{u}(y) \hat{q}_{u}}
$$

Bhattacharyya coefficient values between 0 and 1 , the 
greater the value, the more the similarity. Using the Taylor formula to expand $\hat{\rho}(y)$ at $y_{0}$ :

$$
\begin{aligned}
& \rho(\hat{p}(y), \hat{q})=\frac{1}{2} \sum_{u=1}^{m} \sqrt{\hat{p}_{u}\left(y_{0}\right) \hat{q}_{u}}+\frac{C h}{2} \sum_{i=1}^{n_{k}} w_{i} k\left(\left\|\frac{y-x_{i}}{h}\right\|^{2}\right) \\
& w_{i}=\sum_{u=1}^{m} \sqrt{\frac{\hat{q}_{u}}{\hat{p}_{u}\left(y_{0}\right)}} \delta\left[b\left(x_{i}\right)-u\right]
\end{aligned}
$$

The first item of the formula is a constant. The second can use the Mean shift iterative method to find the maximum value in the neighborhood. According to the Mean Shift calculation method, we calculated recursively at the center of the candidate model at $y_{0}$ in the current frame to obtain the new target center position:

$$
y_{1}=\frac{\sum_{i=1}^{m} x_{i} w_{i} g\left(\left\|\frac{y_{0}-x_{i}}{h}\right\|^{2}\right)}{\sum_{i=1}^{m} w_{i} g\left(\left\|\frac{y_{0}-x_{i}}{h}\right\|^{2}\right)}
$$

Calculating the distance between the old and new position, if $\left\|y_{1}-y_{0}\right\|<\varepsilon$, the operation of the next frame is performed, otherwise calculating the candidate histogram and the mean shift vector at the new location. Then the candidate region will close to the real target area gradually. After several recursive calculations, you can get the real target location.

The target tracking which base on Kalman filter predicts the location information in the next frame by the movement history of the moving target [4]. The Kalman filter model is actually a way to track the target in state space representation, generally includes the target coordinates $X$ and $y$, the movement speed components $v_{x}$ and $v_{y}$, the acceleration elements $a_{x}$ and $a_{y}$. Therefore, an arbitrary point in the state space can be representing as the vector $\left(x, y, v_{x}, v_{y}, a_{x}, a_{y}\right)^{T}$.

We suppose $\Delta t=1$, in the interval the irises move uniformly, the state equation of the irises in the moment $t$ is expressed as:

$$
x_{k}=A x_{k-1}+B u_{k-1}+w_{k-1}
$$

Among them $w_{k-1}$ is system noise. The state transition matrix A can be set to:

$$
A=\left[\begin{array}{llll}
1 & 1 & 0 & 0 \\
0 & 1 & 1 & 0 \\
0 & 0 & 1 & 1 \\
0 & 0 & 0 & 1
\end{array}\right]
$$

In the moment $k$, the measured value $z_{k}$ follows the measurement equation:

$$
z_{k}=H x_{k}+v_{k}
$$

Among them $v_{k}$ is measurement noise. Because $Z_{k}$ only related with position, we can set $H$ is

$$
H=\left[\begin{array}{llll}
1 & 0 & 0 & 0 \\
0 & 1 & 0 & 0
\end{array}\right]
$$

Mean Shift algorithm has the disadvantage of no predictability and is vulnerable to occlusion and background, so we combine the Mean Shift tracking algorithm and the Kalman filtering algorithm, forecast iris position by filter, and Take it as the starting position of the Mean Shift algorithm, then search and match irises. We also proposed improvements. We increased average speed calculation to predict candidate target area, and take it as the speed of the irises when the tracking is interfered by background or the irises are severely blocked. The specific algorithms are below:

1) We calibrate the iris region, and model, then initialize Kalman filter;

2) Using the Kalman filter to predict the position of the iris in the current frame;

3) Using Mean Shift algorithm to calculate the iris position and Bhattacharyya coefficient $\rho_{0}$ in the forecast area, and to calculate the average speed of the irises $\bar{v}_{i}$ in the previous $\mathrm{N}$ frames of the current frame, Where $i$ is the number of frames, if $i<N$, we only calculate the average speed from the first ${ }$ frames;

4) If $\rho_{0}$ is less than the threshold $\rho_{\text {th }}$, we believe that the iris severe occlusion occurs, the results obtained by the Mean Shift algorithm is no longer accurate. At this time, we need to use average speed $\bar{v}_{i}$ to predict iris location, and take it as the forecast to update Kalman filter; otherwise, we take the next step;

5) We calculate the iris speed $v_{i}$ in this moment, and compared with the average speed $\bar{v}_{i}$. When $\left|v_{i}-\bar{v}_{i}\right|$ is bigger than the threshold $\varepsilon_{v}$, we use the average speed $\overline{v_{i}}$ to predict the iris position $y^{\prime}$, and calculate the Bhattacharyya coefficient $\rho^{\prime}$ at $y^{\prime}$. If $\rho^{\prime} \geq \rho_{t h}$, we think that the tracking is interfered by background, $y^{\prime}$ is the true position of the 
iris; otherwise, we think the tracking is not disrupted, continue the search and the match in the next frame.

\section{THE ANALYSIS OF EXPERIMENTAL RESULTS}

This paper first chooses the template matching method remove non-interested region quickly to position iris preliminary. In the tracking algorithm we also choose $\mathrm{H}$ color vector histogram as the characteristic parameter to avoid the interference due to the background and the iris color characteristics are similar. We finished tracking and locating iris coordinate accurately in the case of gaze, squinting, opening the eyes and blink, the tracking results are shown in Figure 4.

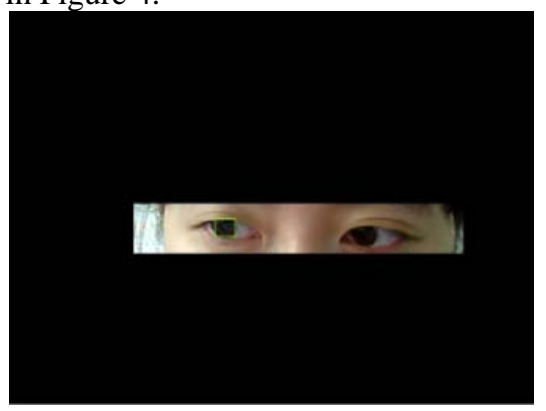

(b) The results when squinting

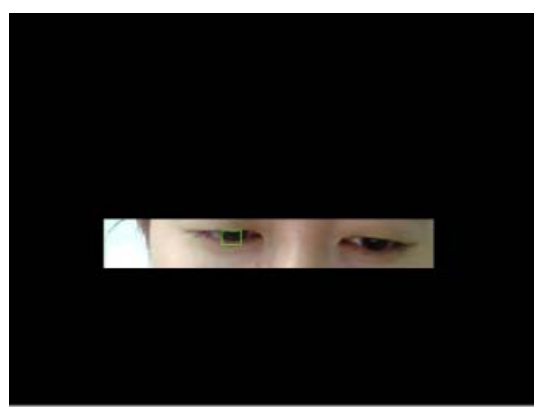

(c) The results when blinking

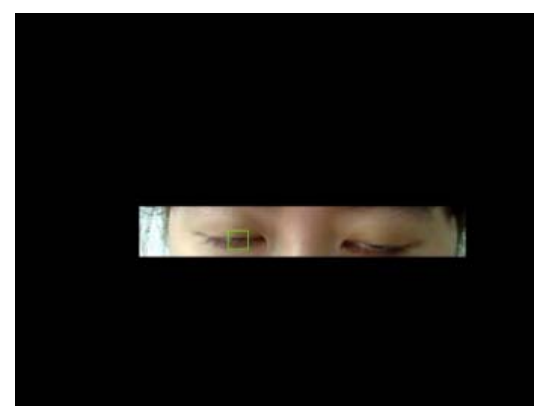

(d) The results when closing the eyes

Figure 4 the iris tracking experimental results

We also put forward the average speed of the target to overcome the disadvantage of losing the target when the

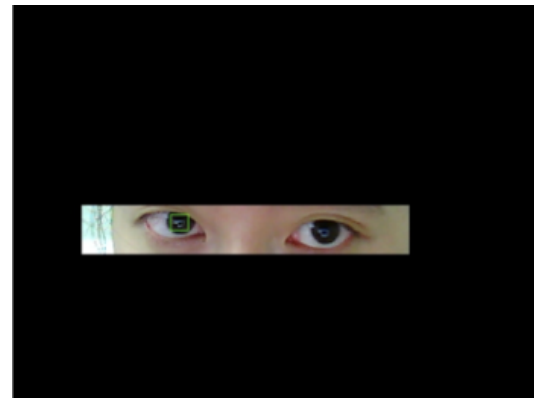

(a) The results when gazing

target is obscured and interference by the background. This method reduced the Mean Shift algorithm iterative times, each frame iterations are less than 5, the average iterations is 2.33. It also accelerates the search speed, takes about $18 \mathrm{~ms}$ per frame, and is able to meet the needs of practical application.

The method still needs to improve. If the face area is too small or partial side and pitching angle is too large, the system will be influence.

\section{REFERENCES}

[1] Shu Mei and Dong Xiu-cheng: "The eye location based on skin color and template matching "submitted to Journal of Computer engineering and application , vol. 45, 2009, pp. 237-239.

[2] Chen Yan-qin and Luo Da-yong: "The eyes of real-time tracking based on Kalman filtering algorithm and MeanShift "submitted to Journal of Pattern recognition and artificial intelligence , vol. 17, 2004, pp. 173-177.

[3] Liu Zong-pu,Zhao Shu-guang and Pan He-xiang: "A new moving target tracking methods base on Kalman prediction and Mean - Shift" submitted to Journal of Optoelectronic technology , vol.29, 2009, pp.30-33.

[4] Saira Saleem Pathan, Ayoub Al-Hamadi and Bernd Michaelis: Proceedings of 2nd International Conference on Computer, Sci. Forum , 2009, pp. 1-6.

[5] National Instruments Corporation, in: NI vision assistant tutorial.USA National Instruments Corporation publishers, 2009. 